\title{
Measured GPS performance under LTE-M in-device interference
}

\author{
Olli Apilo*, Mikko Hiivala*, Atte Kuosmonen ${ }^{\dagger}$, Jani Kallankari ${ }^{\ddagger}$, \\ Hamid Amin ${ }^{\S}$, Mika Lasanen*, Markus Berg ${ }^{\S}$ and Aarno Pärssinen ${ }^{\S}$ \\ *VTT Technical Research Centre of Finland Ltd, Kaitoväylä 1, P.O. Box 1100, FI-90571 Oulu, Finland \\ Email: \{Olli.Apilo, Mikko.Hiivala, Mika.Lasanen\}@vtt.fi \\ ${ }^{\dagger}$ Polar Electro Oy, Professorintie 5, FI-90440 Kempele, Finland, Email: Atte.Kuosmonen@polar.com \\ ¥Verkotan, Elektroniikkatie 17, FI-90590 Oulu, Finland, Email: Jani.Kallankari@ verkotan.com \\ ${ }^{\S}$ Centre for Wireless Communications, University of Oulu, FI-90014 Oulu, Finland \\ Email: \{Hamid.Amin, Markus.Berg, Aarno.Parssinen\}@oulu.fi
}

\begin{abstract}
In this paper we present the measurement results for time-to-fix, position accuracy, and carrier-to-noise ratio of commercial Global Positioning System (GPS) receivers under the in-device interference from an LTE-M transmitter. The laboratory measurement set-up is built using software-defined radio (SDR) platforms to conductively feed emulated GPS L1 signals and LTE-M interference signals to the antenna input of the GPS receivers. The LTE-M interference from second harmonics is accurately modelled taking into account the transmitter activity patterns in different coverage enhancement modes. According to measurements, there are large variations in interference tolerance between different GPS receivers. REC01 was able to tolerate high level of interference during tracking and also in acquisition as long as the interference pulse duration is not too long (tens of milliseconds). REC02 performed clearly worse and tolerated only low levels of LTE-M interference during both acquisition and tracking. The same measurement set-up can be used with any GPS receiver for designing proper isolation and filtering levels for co-existing LTE-M transmitters.
\end{abstract}

\section{INTRODUCTION}

According to recent predictions, approximately 20 billion Internet of Things (IoT) devices will be connected to Internet by 2023 [1]. Many IoT devices require support for mobility, wide coverage, and positioning. For these needs, 3GPP has specified the Cat-M device class, also known as LTE-M, with low cost, extended coverage, optimized power saving, and mobility support. Although 3GPP has also specified positioning methods based on direction of arrival, their performance is not yet satisfactory for all applications [2]. Thus, it is expected that many LTE-M devices will have their positioning based on Global Navigation Satellite Systems (GNSSs) and especially on Global Positioning System (GPS).

The low cost and long battery lifetime of LTE-M modules are achieved by restricting the RF bandwidth to $1.4 \mathrm{MHz}$, having only a single antenna, operating in the half-duplex frequency division duplex (FDD) mode, and restricting the maximum transport block size (TBS) to 1000 bits. This limits the maximum data rates to $300 \mathrm{kbit} / \mathrm{s}$ in downlink (DL) and to $375 \mathrm{kbit} / \mathrm{s}$ in uplink (UL) [3]. When the data rate requirements are low, it is possible to reach over $20 \mathrm{~dB}$ coverage gain over the conventional LTE by repetition transmission. The battery lifetime can exceed 10 years when the data transmission takes place only once per day.

In-device interference occurs when two coexisting radios are simultaneously transmitting and receiving and some of the spectral components of these systems are overlapping or adjacent. The GPS L1 channel at $1575.42 \mathrm{MHz}$ is not directly adjacent to any LTE-M channel as the closest band 21 is more than $100 \mathrm{MHz}$ away [4]. However, the second harmonics of the LTE Tx band 13 at $777-787 \mathrm{MHz}$ are immediately adjacent to the GPS Rx band and can cause interference without proper isolation and filtering. As the transmission time interval (TTI) in LTE is $1 \mathrm{~ms}$ and the GPS bit duration is $20 \mathrm{~ms}$, the in-device interference from typical LTE-M UL transmission is seen as pulse jamming at the GPS receiver [5]. These strong jamming pulses can saturate the analog-to-digital (A/D) converter which completely suppresses the desired GPS signal [6].

The in-device interference problem was studied at 3GPP during Release-10. Based on the study, the RF isolation and filtering cannot provide sufficient interference rejection for all considered coexistence scenarios. For these scenarios, both frequency division multiplexing (FDM) and time division multiplexing (TDM) solutions were proposed [7]. In the FDM solution, the LTE signal is moved away from the frequency of the coexistence signal by performing an inter-frequency handover or restricting the allocation of certain physical resource blocks (PRBs). The inter-frequency handover may not be possible for LTE-M devices because typically LTE-M is supported by only one LTE band per operator over a certain geographical area. However, the base station can allocate narrowbands far from the coexistence signal within the same LTE band. In the TDM solution, the discontinuous reception (DRX) cycle is reconfigured such that a fraction of time is free from LTE transmission and receptions. When the device cannot solve an in-device interference problem by itself, it can request an FDM or TDM solution from the base station using the in-device interference indication message. It is up to base station whether it will follow the requests from the devices.

The amount of interference a GPS receiver can tolerate is obviously implementation-specific, but a typical receiver can tolerate a narrowband interferer that is approximately $40 \mathrm{~dB}$ 
stronger than the received GPS signal [8]. The effect of RF interference from other wireless systems to GPS have been measured and reported in several papers. The second and third harmonics of Digital Video Broadcasting - Terrestrial (DVB-T) on some bands overlap with the GPS receiver bandwidth and can cause interference at the proximity of the broadcasting transmitter stations [9]. The interference from DVB-T results in carrier-to-noise ratio $\left(C / N_{0}\right)$ degradation which may cause problems in lock detection [9], [10]. The effects of interference from the closest LTE band 24 to GPS receivers have been measured and reported in [11]-[13]. The adjacent band LTE interference causes $C / N_{0}$ degradation, increased pseudorange standard deviation, position errors, and increased time-to-fix.

In this paper, we measure the effect of LTE-M interference to acquisition and tracking in commercial GPS receivers. The measurements are done in the laboratory environment where the interfering LTE-M signal together with the emulated GPS signals are conducted to the antenna input of the GPS receivers. The LTE-M interference pattern is accurately modelled in time and frequency domains taking into account the activity patterns of half-duplex LTE-M communications. The GPS performance is reported using the traditional $C / N_{0}$ metric as well as the time for the first fix and the position error statistics, both of which are relevant to GPS users. According to the knowledge of the authors, the measured performance of GPS receivers under the in-device LTE-M interference has not been earlier presented in the scientific literature.

The rest of the paper is organized as follows: The system model for the measurements is described in Section III while the modelling of the LTE-M UL interference signal is described in Section II. The results from the measurement campaign are presented in Section IV. Finally, the conclusions are drawn in Section V.

\section{LTE-M IN-DEVICE INTERFERENCE MODELLING}

In this section, we first describe the time and frequency domain behavior of LTE-M UL transmissions. This is essential in understanding how often and at which frequency the LTE$M$ interference can be seen by the GPS receiver. Then we provide a simple approach for modelling the spectrum of the LTE-M second harmonics and finally we describe the LTE-M interference patterns for the selected scenarios.

\section{A. Time and frequency domain scheduling}

In this study, we consider 4 different physical channels that are all needed for providing DL or UL connectivity during data transmission. DL and UL data are transmitted using physical downlink shared channel (PDSCH) and physical uplink shared channel (PUSCH), respectively. The indications for the upcoming DL data transmission and grants to transmit UL data are sent using machine type communications physical downlink control channel (MPDCCH). If there is no scheduled PUSCH transmission, the acknowledgment for a PDSCH transmission is sent using physical uplink control channel (PUCCH). The following timing relationships have been specified for half-duplex LTE-M [3]:

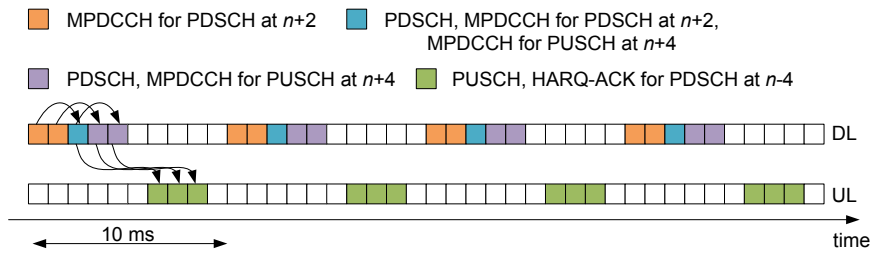

Fig. 1. Half-duplex LTE-M timing example for DL and UL full buffer traffic.

- The indication for a PDSCH transmission at subframe $n$ have to be sent at subframe $n-2$.

- The grant for a PUSCH transmission at subframe $n$ have to be sent at subframe $n-4$.

- The acknowledgement for a PDSCH transmission at subframe $n$ have to be sent at subframe $n+4$.

- The device cannot receive and transmit at the same subframe. There has to be a blank subframe before the link direction is switched from DL to UL or vice versa.

Taking the above restrictions into account, an example LTE$\mathrm{M}$ activity pattern is shown in Fig. 1 where it is assumed that there is full buffer traffic for both directions.

When repetitions are enabled, the same transport block is transmitted multiple times in order to improve coverage. The number of repetitions for each physical channel can be estimated for a given coupling loss and the target data rate [14]. The coupling loss in the link budget analysis is defined as the difference between the conducted power levels measured at the transmitting and receiving antenna ports. Correspondingly, maximum coupling loss (MCL) is defined as the maximum loss in conducted power levels that a system can tolerate and still be operable [3]. MCL $L_{\max }$ can be given as

$$
\begin{aligned}
L_{\max } & =P-S=P-P_{N}-\gamma \\
& =P-N_{0}-F-10 * \log _{10} B-\gamma
\end{aligned}
$$

where $P$ is the transmitted power in $\mathrm{dBm}, S$ is the receiver sensitivity in $\mathrm{dBm}, P_{N}$ is the effective noise power in $\mathrm{dBm}$, $\gamma$ is the minimum tolerated signal-to-noise ratio (SNR) in $\mathrm{dB}, N_{0}$ is the thermal noise density in $\mathrm{dBm} / \mathrm{Hz}, F$ is the noise figure in $\mathrm{dB}$, and $B=180000 N_{\mathrm{PRB}}$ is the transmission bandwith. An example MCL analysis is presented in Table I. We have assumed the each step in TBS index $I_{\text {TBS }}$ corresponds to approx. $1 \mathrm{~dB}$ in the required SNR [15]. Another key assumption is that doubling the number of repetitions $N_{\text {rep }}$ provides $3 \mathrm{~dB}$ coverage gain [14].

An LTE-M device can operate in any LTE system bandwidth but with a limited channel bandwidth of 6 PRBs that is called a narrowband. We assume that the highest narrowband from band 13 is used for scheduling LTE-M PUSCH. This results in the worst case interference from the GPS point of view. PUCCH transmission is mapped to the highest and lowest PRB within band 13 with inter-subframe frequency hopping between them [3]. It is assumed that frequency hopping between repetitions is disabled. 
TABLE I

EXAMPLE MCL ANALYSIS.

\begin{tabular}{|c|c|c|c|c|}
\hline & PUCCH & PUSCH & PDSCH & MPDCCH \\
\hline$N_{\text {PRB }}$ & 1 & 1 & 6 & 6 \\
\hline$N_{\text {rep }}$ & 1 & 1 & 1 & 1 \\
\hline$I_{\text {TBS }}$ & & 2 & 2 & \\
\hline$P$ & 23 & 23 & 36.8 & 36.8 \\
\hline$N_{0}$ & -174 & -174 & -174 & -174 \\
\hline$F[14]$ & 5 & 5 & 9 & 9 \\
\hline $\begin{array}{c}P_{N}=N_{0}+F \\
+10 \log _{10} B\end{array}$ & -116.4 & -116.4 & -104.7 & -104.7 \\
\hline $\begin{array}{c}\gamma[14] \\
S_{i}=P_{N}+\gamma \\
-10 \log _{10} N_{\text {rep }}\end{array}$ & -7.8 & $-6.3+I_{\text {TBS }}$ & $-2+I_{\text {TBS }}$ & -0.7 \\
\hline $\begin{array}{c}L_{\max } \\
=P_{B}-S_{i}\end{array}$ & 147.2 & -120.7 & -104.7 & -105.4 \\
\hline
\end{tabular}

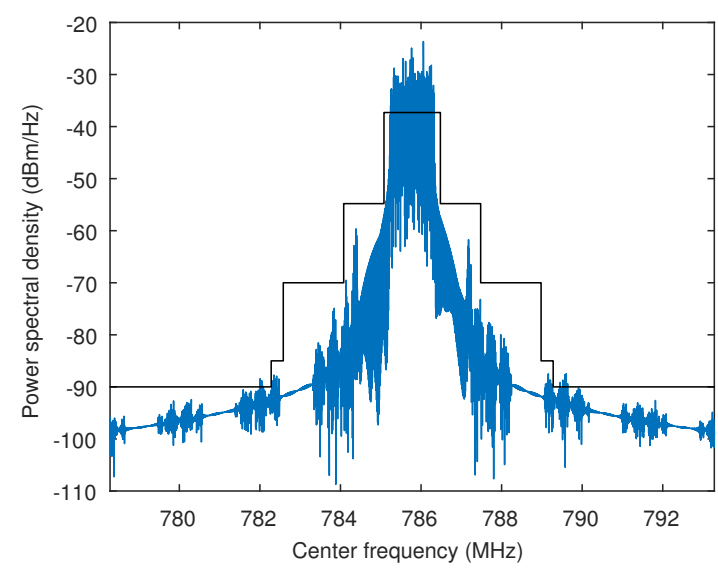

Fig. 2. Up-sampled and filtered LTE UL spectrum at the highest narrowband.

\section{B. Model for second harmonics}

The baseband UL LTE-M signal is generated using the Vienna LTE link level simulator [16]. The signal is up-sampled by factor 16 and pulse shape filtered with the raised root cosine filter with a rolloff factor 0.58 and 81 taps, which results in spectrum shown in Fig. 2. The black line depicts the LTE UL spectral mask that can be occasionally exceeded because the spectral mask is defined as a certain power level measured over a bandwidth of $30 \mathrm{kHz}$ (first out-of-band) or $1 \mathrm{MHz}$ (everywhere else) [4]. Second harmonics can be modelled by squaring and scaling the signal [17]. When we square the signal shown in Fig. 2 and scale its power such that it fits to the LTE UL spurious emission requirements [4], we get the spectrum shown in Fig. 3.

\section{Activity patterns for selected scenarios}

For generating worst case LTE-M activity, we assume that there is full buffer traffic. The varying parameters are $N_{\text {rep }}$, $I_{\mathrm{TBS}}$ and $N_{\mathrm{PRB}}$, which affect the MCL according to Table I and the achievable data rate according to Table 7.1.7.2.1-1 [18]. The selected scenarios are 1) Maximum DL and UL data rate in good channel conditions, 2) Maximum UL data rate in good channel conditions, 3) Coupling loss of $145 \mathrm{~dB}$ with target data rate of $16 \mathrm{kbps}$ (DL) and $8 \mathrm{kbps}$ (UL), and 4) Coupling loss of $155 \mathrm{~dB}$ with target data rate of $500 \mathrm{bps}$. The

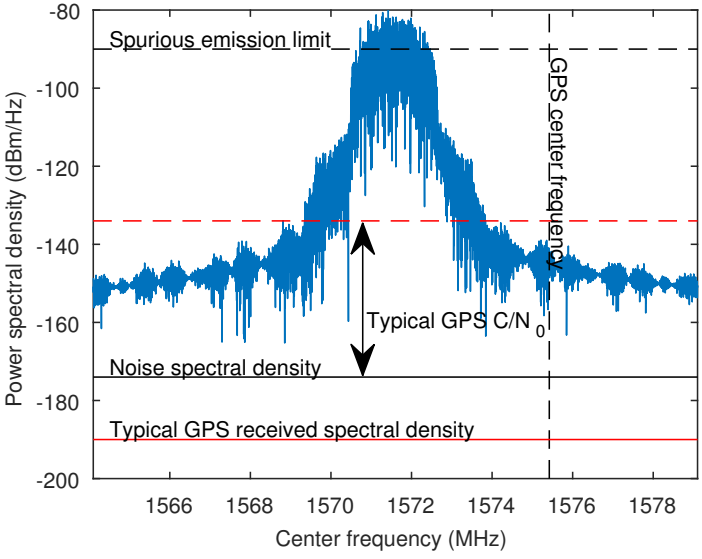

Fig. 3. Example of the LTE UL second harmonics spectrum.

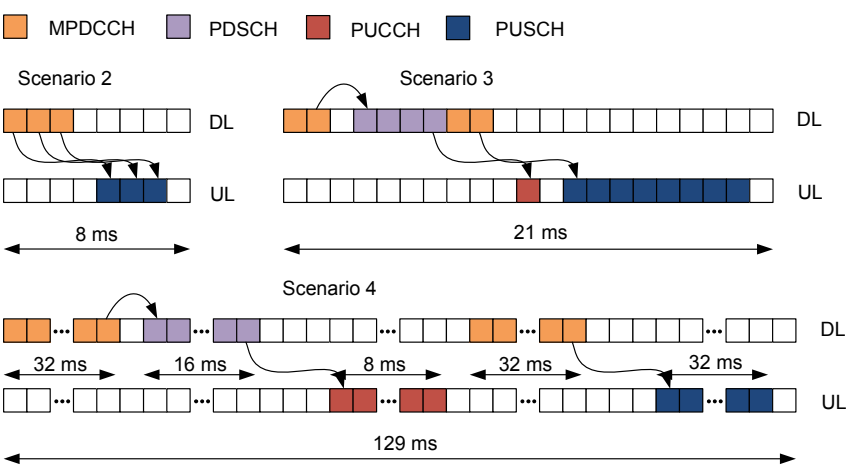

Fig. 4. Timing diagrams for scenarios 2 - 4 .

parameters, resulting timing cycles and data rates are shown in Table II. The timing diagram for scenario 1 is shown in Fig. 1 where we have assumed that MPDCCH can PDSCH can be multiplexed to the same subframe. The timing diagrams for scenarios $2-4$ are shown in Fig. 4.

\section{SYSTEM MODEL}

A block diagram of the measurement system is shown in Fig. 5. The left-most section of the diagram represents the generation of the GPS and LTE-M interference models and offline analysis of the models. The GPS signals are generated using a commercial GNSS simulation software [19]. The software takes a time series of latitude and longitude coordinates at 10 ms intervals as an input and generates the digital IF signals for each satellite for a selected date and time. We convert the signals to baseband IQ samples and digitally combine the signals of the chosen 10 satellites. The bandwidth of the GPS L1 signal is $8.184 \mathrm{MHz}$. We selected to use ideal GPS signals without any impairments or channel models in order to see only the effect of the in-device interference. Furthermore, in the digital combining we selected to scale the signals of the GPS satellites in two different ways: with equal level for all satellites and unequal levels with $3 \mathrm{~dB}$ decrement for each satellite. The first case represents the best case for GPS reception with all 10 satellites visible while the second case 
TABLE II

PARAMETERS, TIMING CYCLES AND DATA RATES FOR THE SELECTED SCENARIOS.

\begin{tabular}{|c|c|c|c|c|c|c|c|c|c|c|c|}
\hline $\begin{array}{c}\text { Coupling } \\
\text { loss (dB) }\end{array}$ & $\begin{array}{c}N_{\text {rep }} \\
\text { MPDCCH }\end{array}$ & $\begin{array}{c}N_{\text {rep }} \\
\text { PUCCH }\end{array}$ & $\begin{array}{c}N_{\text {rep }} \\
\text { PDSCH }\end{array}$ & $\begin{array}{c}N_{\text {rep }} \\
\text { PUSCH }\end{array}$ & $\begin{array}{c}N_{\text {PRB }} \\
\text { PDSCH }\end{array}$ & $\begin{array}{c}N_{\text {PRB }} \\
\text { PUSCH }\end{array}$ & $\begin{array}{c}I_{\text {TBS }} \\
\text { DL }\end{array}$ & $\begin{array}{c}I_{\text {TBS }} \\
\text { UL }\end{array}$ & $\begin{array}{c}\text { Timing } \\
\text { cycle (ms) }\end{array}$ & $\begin{array}{c}\text { Rate } \\
\text { DL (kbps) }\end{array}$ & $\begin{array}{c}\text { Rate } \\
\text { UL (kbps) }\end{array}$ \\
\hline$<127$ & 1 & - & 1 & 1 & 4 & 5 & 13 & 11 & 10 & 300 & 300 \\
\hline$<127$ & 1 & - & - & 1 & - & 5 & - & 11 & 8 & - & 375 \\
\hline 145 & 2 & 1 & 4 & 8 & 6 & 6 & 4 & 1 & 21 & 19.4 & 9.9 \\
\hline 155 & 32 & 8 & 16 & 32 & 6 & 2 & 0 & 2 & 129 & 1.2 & 0.6 \\
\hline
\end{tabular}

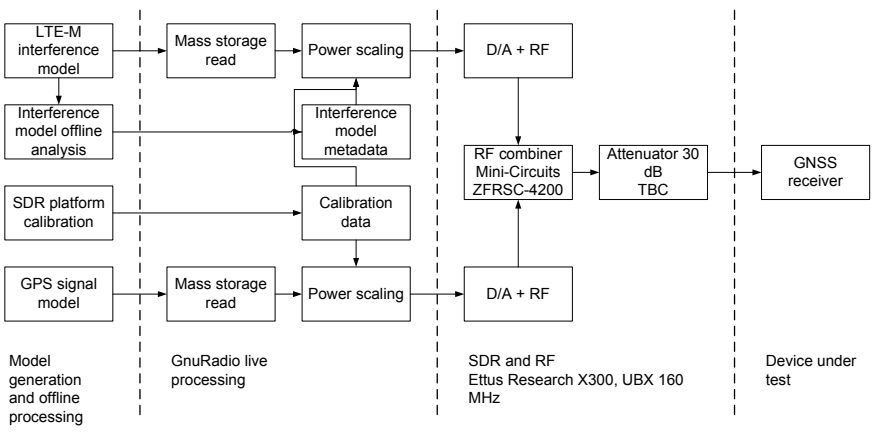

Fig. 5. Block diagram of measurement set-up.

TABLE III

REPORTED $C / N_{0}$ LEVELS WITH NO INTERFERENCE.

\begin{tabular}{|c|c|c|c|c|}
\hline \multirow{2}{*}{ Satellite PRN id } & \multicolumn{2}{|c|}{ REC01 } & \multicolumn{2}{c|}{ REC02 } \\
\cline { 2 - 5 } & Equal & Unequal & Equal & Unequal \\
\hline 5 & 40 & 40 & 43 & 42 \\
\hline 9 & 40 & 37 & 43 & 39 \\
\hline 16 & 40 & 34 & 43 & 36 \\
\hline 20 & 40 & 31 & 43 & 33 \\
\hline 21 & 40 & 28 & 43 & 30 \\
\hline 23 & 40 & 25 & 43 & 27 \\
\hline 26 & 40 & 22 & 43 & - \\
\hline 27 & 40 & 19 & 43 & - \\
\hline 29 & 40 & 16 & 43 & - \\
\hline 31 & 40 & - & 43 & - \\
\hline
\end{tabular}

represents GPS reception under bad signal conditions. For the equal level case the GPS sum signal power at the receiver input is $-113 \mathrm{dBm}$, which corresponds to $-179 \mathrm{dBm} / \mathrm{Hz}$ and -182 $\mathrm{dBm} / \mathrm{Hz}$ measured over 4 and $8 \mathrm{MHz}$ bandwidths, respectively. For the unequal level case the corresponding GPS sum signal power is $-122 \mathrm{dBm}(-188 \mathrm{dBm} / \mathrm{Hz}$ and $-191 \mathrm{dBm} / \mathrm{Hz})$. The $C / N_{0}$ levels with no interference are shown in Table III. It can be seen that REC02 reports higher $C / N_{0}$ than REC01, which can indicate that REC02 uses higher bandwidth for the GPS reception. However, as the data sheets of the receivers do not show the reception bandwidths, we cannot confirm this. The in-device interference from LTE-M transmission is modelled according to the process described in detail in Section II. The GPS and interference signal models are stored in the measurement system mass storage as 8-bit IQ sample files.

We use USRP X300 software-defined radio (SDR) platforms from Ettus Research to generate the RF signals from the models. The runtime control of the USRP platforms is implemented using the UHD [20] and GNU Radio software frameworks [21]. Using the GNU Radio framework, we imple- mented efficient mass storage reader and real time controllable digital power scaling blocks for the system. The power scaling blocks are used to set the chosen power levels for the GPS and interference signals during the measurements. The FPGAcontrolled hardware in the USRP platforms converts the digital signals to analog and performs the mixing to RF. We use two separate platforms to generate the GPS signal and interference signal due to their differing bandwidths and sampling frequencies. As mentioned earlier, the GPS signal bandwidth is $8.184 \mathrm{MHz}$ and the 16 times oversampled bandwidth of the LTE-M interference is $30.72 \mathrm{MHz}$. We use an RF combiner to combine the signals and feed them to the GPS receiver under test through a $30 \mathrm{~dB}$ attenuator. The RF attenuator enables the software and SDR platform chain to operate at a higher signal level for better resolution at the digital-to-analog converters and lowers the noise floor of the system.

\section{Measurement Results}

We present the average time-to-fix during acquisition and the average position error and $C / N_{0}$ during tracking for two commercial GPS receivers REC01 and REC02. All results were extracted from the NMEA data output by the receivers, using the GPS simulator input coordinates as the reference track for positioning results. Both receivers were configured to $1 \mathrm{~Hz}$ operating mode, meaning they output position and satellites-in-view information once per second. Except for disabling any $C / N_{0}$ and satellite elevation masks, the receivers were tested using factory default configuration. The in-device interference from LTE-M is modelled according to the four scenarios described in Section II. Additionally as a reference, we measure the "alwaysOn" scenario that corresponds to an extreme case where the interference from LTE-M is always present. The number of test iterations for acquisition is 20 . The start of the LTE-M interference cycle with respect to the received GPS signal is randomly drawn for each iteration. For tracking, measurements are done for a duration of $363.3 \mathrm{~s}$.

According to 3GPP requirements [4], the maximum level for the LTE interference power at the second harmonics frequency is $-30 \mathrm{dBm}$ measured over $1 \mathrm{MHz}$ bandwidth (-90 $\mathrm{dBm} / \mathrm{Hz}$ ). When we assume that all 6 PRBs from the highest narrowband of band 13 are scheduled, the center frequency for the second harmonics is at $1571.56 \mathrm{MHz}$ (see Fig. 3). The corresponding interference at GPS center frequency is $61 \mathrm{dBm}(-127 \mathrm{dBm} / \mathrm{Hz})$ and $-33 \mathrm{dBm}(-102 \mathrm{dBm} / \mathrm{Hz})$ for 4 and $8 \mathrm{MHz}$ Rx bandwidths, correspondingly.

The average time-to-fix as a function of LTE interference power are shown in Figs. 6 and 7 for equal and unequal 


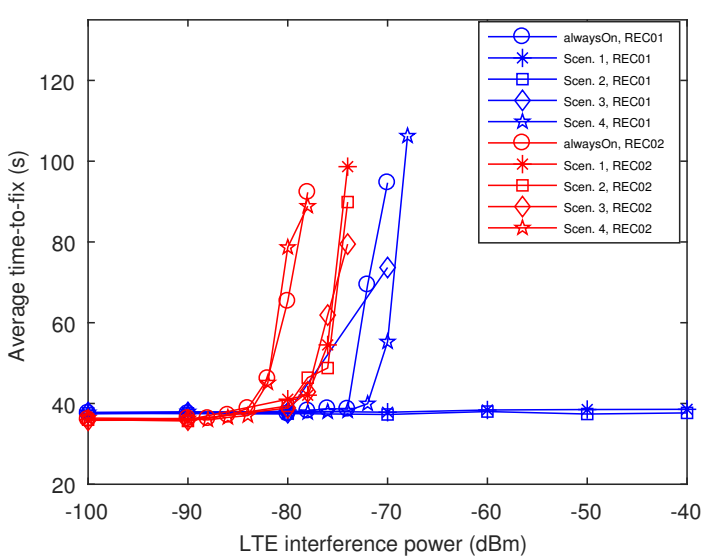

Fig. 6. Average time-to-fix as a function of LTE interference power for equal satellite attenuation.

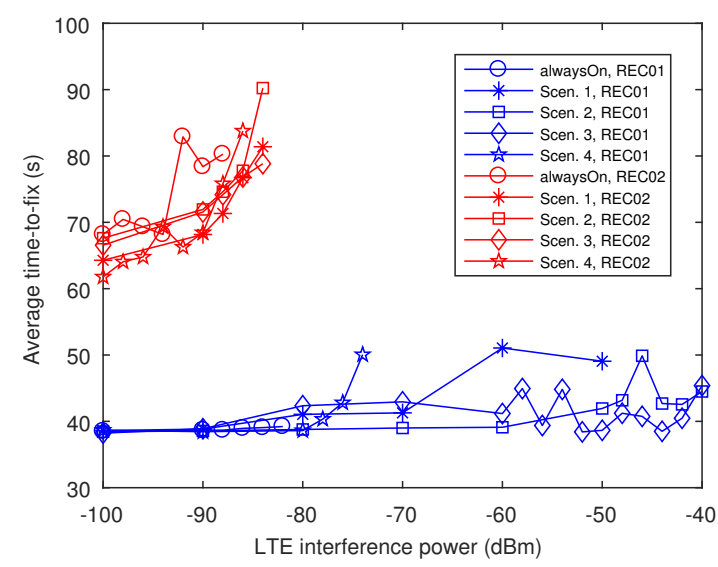

Fig. 7. Average time-to-fix as a function of LTE interference power for unequal satellite attenuation.

satellite attenuation, respectively. The cases where the fix success rate is less than $90 \%$ are excluded from the figures. Under equal satellite attenuation, REC01 has no performance degradation for Scenarios 1 and 2. For Scenarios 3 and 4, the normal performance is achieved when the LTE-M interference is below $-70 \mathrm{dBm}$. The REC02 time-to-fix starts to increase when the LTE-M interference is $-82 \mathrm{dBm}$ for Scenario 4 and $78 \mathrm{dBm}$ for other scenarios. Under bad channel conditions, i.e. with unequal satellite attenuation, it is more relevant to check when the fix probability goes below $90 \%$. REC01 is able get the fix for Scenarios 1 and 2 at all interference levels. For Scenario 4, REC01 does not get the fix at LTE-M interference levels above $-68 \mathrm{dBm}$. The LTE-M interference threshold for getting the fix for REC02 is $-85 \mathrm{dBm}$. Based on Figs. 6 and 7, we can conclude that REC01 tolerates well interference during acquisition as long as the duration of the interference is short ( $<10 \mathrm{~ms}$ ). Surprisingly, REC02 performs much worse and tolerates only low levels of LTE-M interference (around -80 $\mathrm{dBm}$ ). One potential explanation could be that REC02 is using wider Rx bandwidth with no effective interference mitigation.

The average positioning error under tracking is shown in Figs. 8 and 9 for equal and unequal satellite attenuations,

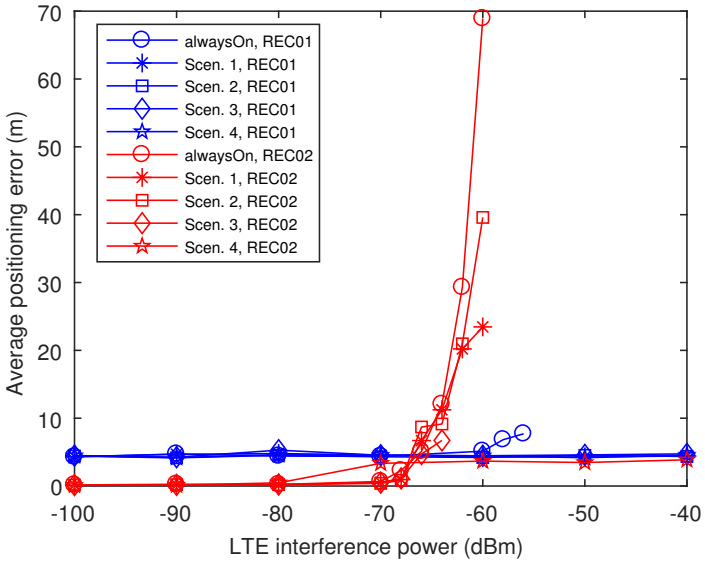

Fig. 8. Average positioning error as a function of LTE interference power for equal satellite attenuation.

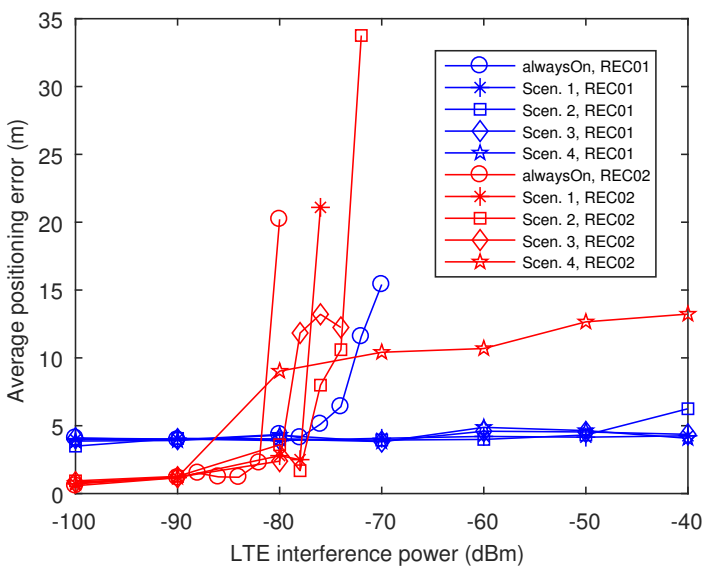

Fig. 9. Average positioning error as a function of LTE interference power for unequal satellite attenuation.

respectively. REC01 is able to keep approximately the same accuracy under all the half-duplex LTE-M interference scenarios. REC02 provides accurate position estimates for low LTE-M interference levels $(\leq-70 \mathrm{dBm}$ in equal and $\leq-80$ $\mathrm{dBm}$ unequal satellite attenuation, respectively). For high interference levels $(>-60 \mathrm{dBm}$ (equal) and $>-70 \mathrm{dBm}$ (unequal)), REC02 is not able to provide position estimates in Scenarios 1-3. In Scenario 4, REC02 seems to be able to utilize the long periods without interference for tracking and does not loose the fix even at high interference.

To illustrate the effect of LTE-M interference to the $C / N_{0}$ levels, the average $C / N_{0}$ for the strongest satellite under tracking is shown in Fig. 10. REC02 reports higher $C / N_{0}$ for low interference levels than REC01. At high interference levels, the larger assumed bandwidth of REC02 includes more interference power close to the peak of the LTE-M second harmonics signal and $C / N_{0}$ is clearly degraded. REC02 reports higher $C / N_{0}$ level for scenario 4 at high LTE-M interference $(\geq-70 \mathrm{dBm})$ than for other scenarios. This may be due to short averaging time that causes some of the $C / N_{0}$ reports to be calculated without any interference. 


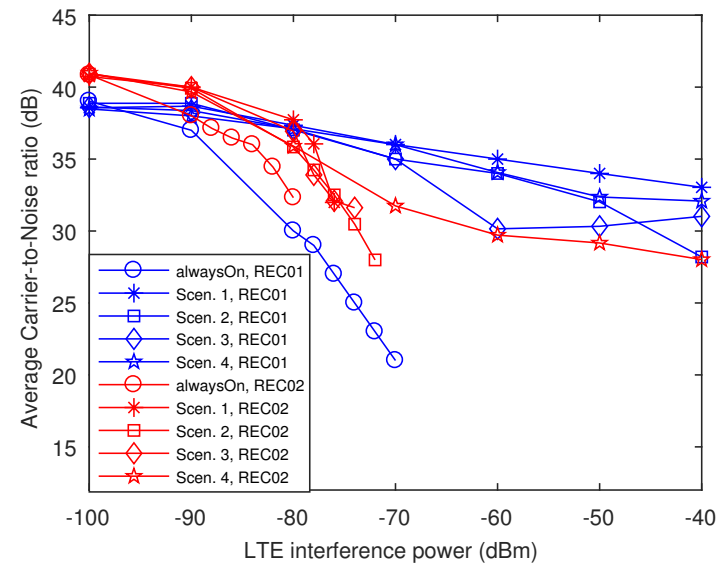

Fig. 10. Average $C / N_{0}$ of the strongest satellite as a function of LTE interference power for unequal satellite attenuation.

Based on the measurement results, we can conclude that REC01 can tolerate LTE-M interference in most of the normal usage scenarios. Only scenario 4, which corresponds to extreme coverage scenarios with high number of repetitive UL transmissions, it can tolerate at maximum $-70 \mathrm{dBm}$ LTE$M$ interference power if longer time-to-fix is allowed. This means that approx. $40 \mathrm{~dB}$ isolation and/or filtering needs to be designed. REC02 can tolerate $-85 \mathrm{~dB}$ ( $55 \mathrm{~dB}$ isolation) and $-80 \mathrm{~dB}$ (50 dB isolation) LTE-M interference during acquisition and tracking, respectively. The variation in measurement results between REC01 and REC02 is surprisingly large. Although both receivers appear us as black boxes without revealing their internal design, the results seem to indicate that REC02 is using wider reception bandwidth. When interpreting the results, it should be kept in mind that we have selected the worst case scenario for LTE-M devices, i.e. LTE band 13 and the highest frequency narrowband. Most of the band 13 in-device coexistence issues between LTE-M and GPS can be avoided by restricting the resource allocation for LTE-M devices to narrowbands closer to the lower edge of band 13 .

\section{Conclusions}

In this paper, we have measured how two commercial GPS receivers, REC01 and REC02, perform under the worst case in-device interference from the second harmonics of an LTE$\mathrm{M}$ transmitter. The performance indicators were the time-tofix during acquisition and $C / N_{0}$ and position accuracy during tracking. An important part of this work was the design and set-up of the test environment that enables measuring the interference behavior of GPS receivers in controlled conditions. The measurements were done by conducting both the emulated GPS signals and interfering LTE-M signal to the antenna input of the GPS receivers. The LTE-M interference were accurately modelled taking into account the activity cycles of half-duplex LTE-M UL transmitters.

REC01 performed well both in acquisition and tracking in most of the interference scenarios. Only in the extreme coverage scenario with repetitive LTE-M transmissions, REC01 is not able to perform in an acceptable way when LTE-M interference power is more than $-70 \mathrm{dBm}$. REC02 performed clearly worse and tolerated only $-85 \mathrm{dBm}$ and $-80 \mathrm{dBm}$ LTE$\mathrm{M}$ interference power during acquisition and tracking, respectively. As the maximum allowed LTE-M second harmonics transmitted power is $-30 \mathrm{dBm}$, the interference signal needs to be attenuated up to $55 \mathrm{~dB}$ by filtering and isolation. The variation in results between REC01 and REC02 was surprisingly large. It can be explained by different $\mathrm{Rx}$ bandwidth and different interference mitigation approaches. The results demonstrate that knowledge of the tolerated interference levels at GPS receivers is very useful for RF and antenna design of co-located LTE-M transmitter in order to avoid performance degradation or too conservative design choices.

\section{ACKNOWLEDGMENT}

The work was done in the Hilla WERME project that is partly funded by Business Finland.

\section{REFERENCES}

[1] "Ericsson mobility report," White Paper, Ericsson, Nov. 2017.

[2] X. Lin et al., "Positioning for the Internet of Things: A 3GPP perspective," IEEE Commun. Mag., vol. 55, no. 12, Dec. 2017.

[3] O. Liberg, M. Sundberg, Y.-P. E. Wang, J. Bergman, and J. Sachs, Cellular Internet of Things: Technologies, Standards, and Performance. London, UK: Academic Press, 2018.

[4] User Equipment (UE) radio transmission and reception, 3GPP TS 36.101, V13.9.0, Sep. 2017.

[5] Z. Hu et al., "Interference avoidance for in-device coexistence in 3GPP LTE-Advanced: Challenges and solutions," IEEE Commun. Mag., vol. 50, no. 11, pp. 60-67, Nov. 2012.

[6] C. Hegarty et al., "Suppression of pulsed interference through blanking," in Proc. IAIN World Congress, San Diego, CA, Jun. 2000.

[7] Study on signalling and procedure for interference avoidance for indevice coexistence, 3GPP TR 36.816, V11.2.0, Dec. 2011.

[8] M. S. Braasch and A. J. Van Dierendonck, "GPS receiver architectures and measurements," Proc. IEEE, vol. 87, no. 1, pp. 48-64, Jan. 1999.

[9] B. Motella, M. Pini, and F. Dovis, "Investigation on the effect of strong out-of-band signals on global navigation satellite systems receivers," GPS Solut., vol. 12, no. 2, pp. 77-86, Mar. 2008.

[10] M. Wildemeersch, A. Rabbachin, E. Cano, and J. Fortuny, "Interference assessment of DVB-T within the GPS L1 and Galileo E1 band," in Proc. ESA NAVITEC, Noordwijk, The Netherlands, Dec. 2010.

[11] M. Rao, C. O'Driscoll, D. Borio, and J. Fortuny, "LightSquared effects on estimated $\mathrm{C} / \mathrm{N}_{0}$, pseudoranges and positions," GPS Solut., vol. 18, no. 1, pp. 1-13, Jan. 2014.

[12] W. F. Young et al., "LTE impacts on GPS," NASCTN, NIST Technical Note 1952, Feb. 2017.

[13] S. Mackey et al., "DOT GPS adjacent band compatibility assessment test results," in Proc. International Technical Meeting of The Institute of Navigation, Monterey, CA, Jan. 2017, pp. 637-661.

[14] "Coverage analysis of LTE-M Category-M1," White Paper, 3GPP, Jan. 2017.

[15] S. Park, R. C. Daniels, and R. W. Heath, "Optimizing the target error rate for link adaptation," in Proc. IEEE GLOBECOM, San Diego, CA, Dec. 2015.

[16] M. Rupp, S. Schwarz, and M. Taranetz, The Vienna LTE-Advanced Simulators: Up and Downlink, Link and System Level Simulation. Singapore: Springer, 2016.

[17] R. E. Ziemer and W. H. Tranter, Principles of Communication Systems, Modulation, and Noise, 4th ed. New York: John Wiley \& Sons, 1995.

[18] Physical layer procedures, 3GPP TS 36.213, V13.5.0, Mar. 2017.

[19] ReGen software for GNSS RF simulator. [Online]. Available: http://www.ip-solutions.jp/regen.html

[20] The USRP Hardware Driver Repository. [Online]. Available: https://github.com/EttusResearch/uhd

[21] GNU Radio. [Online]. Available: https://github.com/gnuradio/gnuradio 